\title{
Classification Seheme
}

1. Structure of Crystalline Solids

1.1 Perfectly Periodic Structure

1.2 Solid-State Phase Transformations

1.3 Alloys. Metallurgy

1.4 Microstructure (Magnetic Domains See 18; Ferroelectric Domains See 14.4.1)

1.5 Films

1.6 Surfaces

2. Non-Crystalline State

3. Crystal Growth

4. Bonding Properties

5. Mössbauer Spectroscopy

6. Lattice Dynamics. Phonons

7. Acoustic Properties

8. Thermal Properties

9. Diffusion

10. Defect Properties (Irradiation Defects See 11)

10.1 Metals

10.2 Non-Metals

11. Irradiation Effects (X-Ray Diffraction Investigations See 1 and 10)

12. Mechanical Properties (Plastic Deformations See 10)

12.1 Metals

12.2 Non-Metals

13. Electron States

13.1 Band Structure

13.2 Fermi Sutfaces

13.3 Surface and Interface States

13.4 Impurity and Defect States

13.5 Elementary Excitations (Phonons See 6)

13.5.1 Excitons

13.5.2 Plasmons

13.5.3 Polarons

13.5.4 Magnons

14. Electrical Properties. Transport Phenomena

14.1 Metals. Semi-Metals

14.2 Superconductivity. Superconducting Materials and Devices

14.3 Semiconductors

14.3.1 Films

14.3.2 Surfaces and Interfaces

14.3.3 Devices. Junctions (Contact Problems See 14.3.4)

14.3.4 High-Field Phenomena, Space-Charge Effects, Inhomogeneities, Injected Car-

14.4 Dielectrics riers (Electroluminescence See 20.3; Junctions See 14.3.3)

14.4.1 Ferroelectrics

15. Thermoelectric and Thermomagnetic Properties

16. Photoconductivity. Photovoltaic Effects

17. Emission of Electrons and Ions

17.1 Field Emission Microscope Investigations

18. Magnetic Properties

18.1 Paramagnetic Properties

18.2 Ferromagnetic Properties

18.2.1 Ferromagnetic Films

18.3 Ferrimagnetic Properties

18.4 Antiferromagnetic Properties 